Europium doped gallium oxide nanostructures for room temperature luminescent photonic devices

This article has been downloaded from IOPscience. Please scroll down to see the full text article.

2009 Nanotechnology 20115201

(http://iopscience.iop.org/0957-4484/20/11/115201)

View the table of contents for this issue, or go to the journal homepage for more

Download details:

IP Address: 147.96.14.16

The article was downloaded on 20/02/2013 at 18:57

Please note that terms and conditions apply. 


\title{
Europium doped gallium oxide nanostructures for room temperature luminescent photonic devices
}

\author{
E Nogales ${ }^{1}$, B Méndez ${ }^{1}$, J Piqueras ${ }^{1}$ and J A García ${ }^{2}$ \\ ${ }^{1}$ Departamento de Física de Materiales, Facultad de Ciencias Físicas, \\ Universidad Complutense de Madrid, Madrid 28040, Spain \\ ${ }^{2}$ Departamento de Física Aplicada II, Apartado 644, Universidad del País Vasco, 48080, Spain \\ E-mail: emilio.nogales@ fis.ucm.es
}

Received 17 November 2008, in final form 3 February 2009

Published 24 February 2009

Online at stacks.iop.org/Nano/20/115201

\begin{abstract}
Cathodoluminescence and photoluminescence techniques have been used to investigate room temperature light emission from $\beta-\mathrm{Ga}_{2} \mathrm{O}_{3}$ :Eu nanostructures, which were obtained by two methods. In one of them, a mixture of $\mathrm{Ga}_{2} \mathrm{O}_{3} / \mathrm{Eu}_{2} \mathrm{O}_{3}$ powders was used as precursor material and annealed under an argon flow. In the other one, undoped $\beta-\mathrm{Ga}_{2} \mathrm{O}_{3}$ nanostructures were first obtained by thermal oxidation of metallic gallium and europium was subsequently incorporated by a diffusion process. Room temperature luminescence at $610 \mathrm{~nm}$ due to $\mathrm{Eu}^{3+}$ intraionic transitions from $\beta-\mathrm{Ga}_{2} \mathrm{O}_{3}$ :Eu has been observed. Waveguiding of this red emitted light through the structures was shown.
\end{abstract}

(Some figures in this article are in colour only in the electronic version)

Semiconductor nanowires and nanobelts exhibit particular physical properties, very interesting for novel photonic nanodevices. These nanostructures may play different roles, such as light emitters [1, 2], waveguides [3, 4] or light detectors $[5,6]$. In the last years, an intense activity has been carried out on nanostructures based on binary oxide semiconductors, such as $\mathrm{ZnO}$ or $\mathrm{SnO}_{2}$ among others [7]. In particular, monoclinic $\beta-\mathrm{Ga}_{2} \mathrm{O}_{3}$ is a promising material for optoelectronic applications and the physical properties of thin films of this material have been the subject of study by several groups [8-12]. The main reasons for the strong interest on this material are its high thermal and chemical stability as well as its wide band gap, around $5 \mathrm{eV}$, while preserves n-type conductivity attributed in most of the cases to native oxygen vacancies [9, 13]. In fact, gallium oxide is the transparent conductive oxide with widest transparency range and its fairly high refractive index, around 2 in the visible-UV range, makes this oxide of interest for waveguiding applications. Its wide band gap enables to modify the luminescence properties by the incorporation of suitable ions in the $\mathrm{Ga}_{2} \mathrm{O}_{3}$ lattice. In particular, doping of $\mathrm{Ga}_{2} \mathrm{O}_{3}$ with optically active ions, such as rare earth (RE) ions, allows the fabrication of devices based on this material which can emit light in different wavelengths spanning from the UV, all the way through the visible to the IR ranges. Although the intraionic emissions of the RE ions are usually stable and almost independent on the matrix, there are problems in rare earth doping of bulk semiconductors due to the difficulty to achieve effective concentrations or the need of further thermal treatments to activate the RE ion luminescence [14]. Some of these problems can be overcome in the case of nanostructures during the fabrication process.

There is a number of works related to undoped gallium oxide nanostructures with different shapes, e.g. nanowires, nanorods and nanobelts, obtained following different routes [7] and, in particular, thermal treatments involving vapor-solid (VS) or vapor-liquid-solid (VLS) mechanisms have been used for the growth of these nanostructures $[11,15]$. Several works on light emitting doped gallium oxide have appeared during the last years. $\mathrm{Eu}^{3+}[16]$ and $\mathrm{Cr}^{3+}[8]$ ions were introduced in bulk gallium oxide, while $\mathrm{Er}^{3+}$ and $\mathrm{Cr}^{3+}$ ions have been introduced into gallium oxide nanostructures $[4,17,18]$. Waveguiding behavior of $\beta-\mathrm{Ga}_{2} \mathrm{O}_{3}$ nanowires doped with optically active ions, $\mathrm{Cr}^{3+}$ or $\mathrm{Er}^{3+}$, has been reported [4].

In this paper, we demonstrate the growth of Eu doped gallium oxide nanowires by two synthesis routes, which enable to grow the nanostructures and to activate the europium ions. 

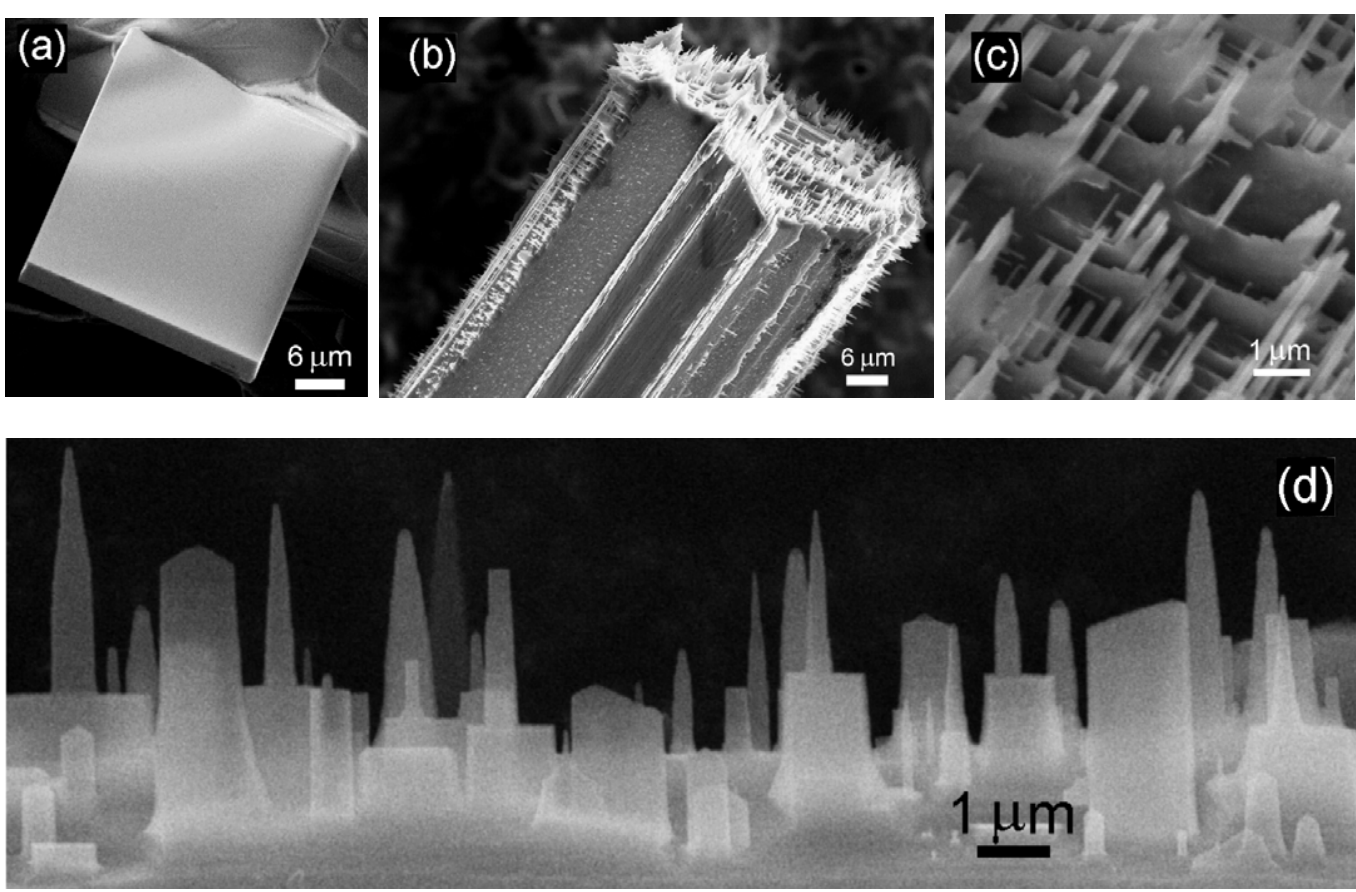

Figure 1. SEM images of Eu doped $\beta-\mathrm{Ga}_{2} \mathrm{O}_{3}$ structures in samples (a) M1 and (b)-(d) M2.

The effective incorporation of $\mathrm{Eu}^{3+}$ is demonstrated by cathodoluminescence (CL) and photoluminescence (PL) measurements. We characterize the red luminescence band related to the characteristic intraionic transition of the rare earth ion and assess the waveguiding of such luminescence.

Two different procedures were used to grow the nanostructures. In one of them a mixture of high purity $90 \mathrm{wt} \% \mathrm{Ga}_{2} \mathrm{O}_{3}$ and $10 \mathrm{wt} \% \mathrm{Eu}_{2} \mathrm{O}_{3}$ powders was milled for $30 \mathrm{~h}$, pressed into a pellet and subsequently annealed under an argon flow at high temperatures either in a single treatment at $1500^{\circ} \mathrm{C}$ for $15 \mathrm{~h}$ (sample M1) or a double treatment, at $1500{ }^{\circ} \mathrm{C}$ for $10 \mathrm{~h}$ and $1350^{\circ} \mathrm{C}$ for $5 \mathrm{~h}$ (sample M2). A similar process was used in [18] to obtain erbium doped gallium oxide micro and nanostructures. A two steps procedure starting from a different precursor has also been used: first, nanowires and nanobelts have been grown, as will be shown in figure 2 , by thermal oxidation of metallic $\mathrm{Ga}$ on a $\mathrm{Ga}_{2} \mathrm{O}_{3}$ pellet under an $\mathrm{Ar}$ flow at $1150{ }^{\circ} \mathrm{C}$; in the second step, the sample with nanostructures was annealed at $1500{ }^{\circ} \mathrm{C}$ in the presence of $\mathrm{Eu}_{2} \mathrm{O}_{3}$ in order to achieve $\mathrm{Eu}$ doping by diffusion (sample D). All samples M1, M2 and D presented micro and nanostructures doped with luminescent europium ions. For comparison, we have obtained a reference $\beta-\mathrm{Ga}_{2} \mathrm{O}_{3}$ :Eu sample by annealing a $\mathrm{Ga}_{2} \mathrm{O}_{3}$ pellet at $1500^{\circ} \mathrm{C}$ in the presence of $\mathrm{Eu}_{2} \mathrm{O}_{3}$ powder to obtain diffusion of the rare earth into its surface (sample R). No micro or nanostructures were obtained in this sample due to differences in the argon flow. The crystal properties of the samples were studied by $\mathrm{x}$-ray diffraction (XRD), transmission electron microscopy (TEM), high resolution-TEM (HRTEM) and selected area electron diffraction (SAED). The morphology of the structures was characterized by scanning electron microscopy (SEM) in a Leica 440 Stereoscan microscope. A Bruker energy dispersive $\mathrm{x}$-ray microanalysis (EDX) system was used for the compositional studies. The luminescence measurements were performed with a cathodoluminescence (CL) system in a SEM, working at $15-20 \mathrm{keV}$ and $1-5 \mathrm{nA}$, or a microphotoluminescence $(\mu$-PL) system included in an Olympus optical microscope with a $\mathrm{Hg}$ lamp.

SEM secondary electron images of the structures obtained by the first procedure are shown in figure 1(a), sample M1, and in figures 1(b)-(d), sample M2. The growth of nanostructures on certain faces of the microstructures occurs after the second thermal treatment, at lower temperature, in sample M2. In particular, nanowires with lateral dimensions ranging from tens to hundreds of nanometers are obtained in this way. A similar result was observed in erbium doped $\beta-\mathrm{Ga}_{2} \mathrm{O}_{3}$ [18].

Figure 2 shows SEM images of the micro and nanostructures obtained from metallic gallium by annealing at different temperatures and Ar flow. Structures such as nanoribbons (figure 2(a)), nanorings (figure 2(b)), nanoneedles (figure 2(c)) and nanorods are observed. The subsequent annealing at $1500{ }^{\circ} \mathrm{C}$ in the presence of $\mathrm{Eu}_{2} \mathrm{O}_{3}$ does not significantly change the morphology of the structures, as figure 2(d) of sample D shows. TEM images, figures 2(e) and (f), demonstrate the high crystal quality of the nanostructures grown in this way. Figure 2(e) shows a nanowire grown in the (001) direction with a lateral grown nanosheet.

Figure 3 shows the EDX spectrum of the nanostructures obtained by annealing the powder mixture (sample M1). Similar results are obtained in samples M2, D and R. Quantitative microanalysis yields a Eu concentration of around 1 at.\%. A similar quantitative value was obtained in the doped nanostructures from sample D.

XRD measurements performed on samples M1 and M2 reveal the presence of $\mathrm{Eu}_{3} \mathrm{Ga}_{5} \mathrm{O}_{12}$, europium gallium garnet 

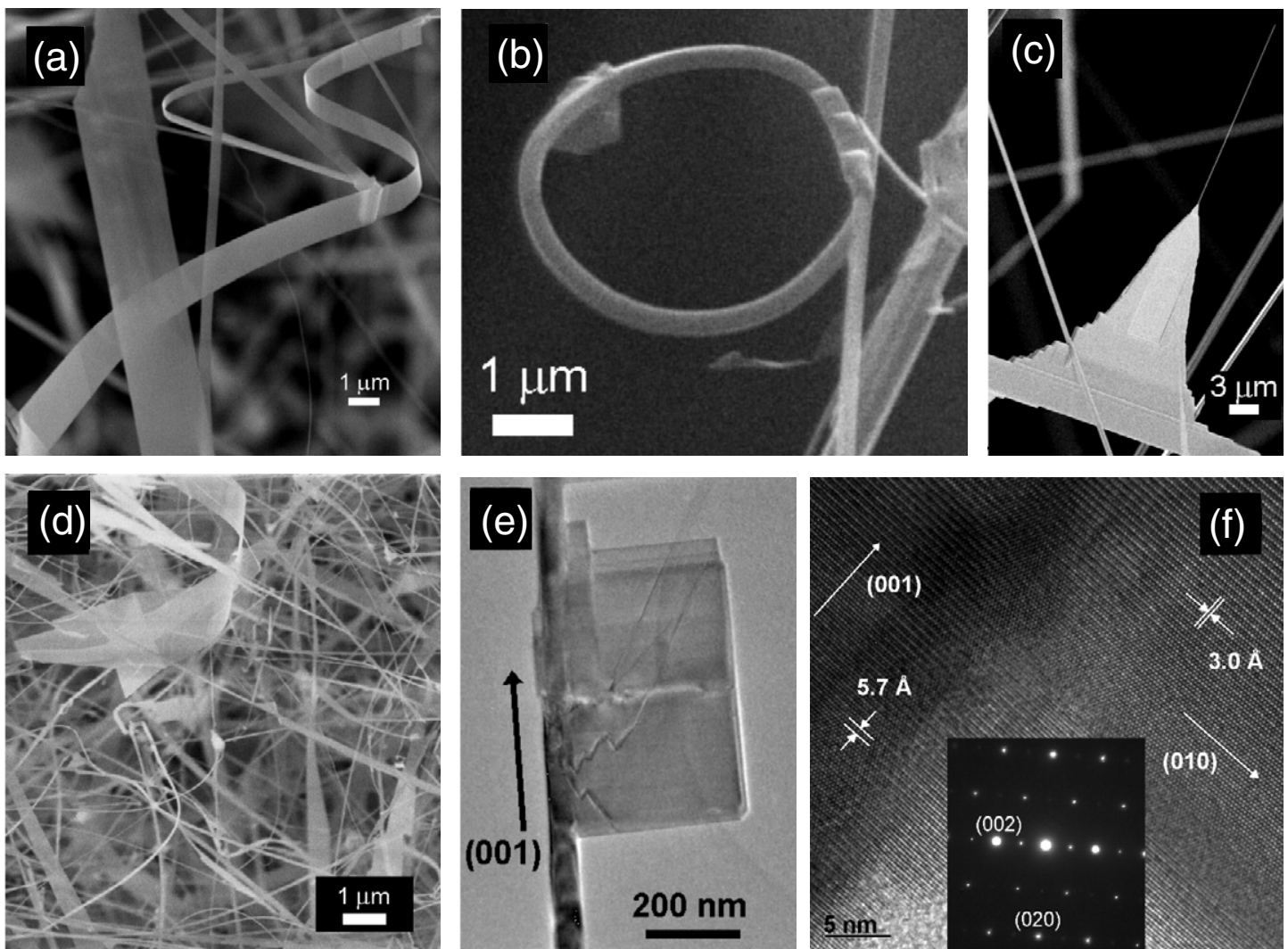

Figure 2. SEM images of (a)-(c) undoped nanowires obtained from metallic gallium and (d) Eu doped $\beta-\mathrm{Ga}_{2} \mathrm{O}_{3}$, sample D. The morphology of the structures is not significantly affected by the $1500^{\circ} \mathrm{C}$ annealing for Eu doping. (e) TEM and (f) HRTEM (inset: SAED) showing that the nanowires of sample D are single crystalline.

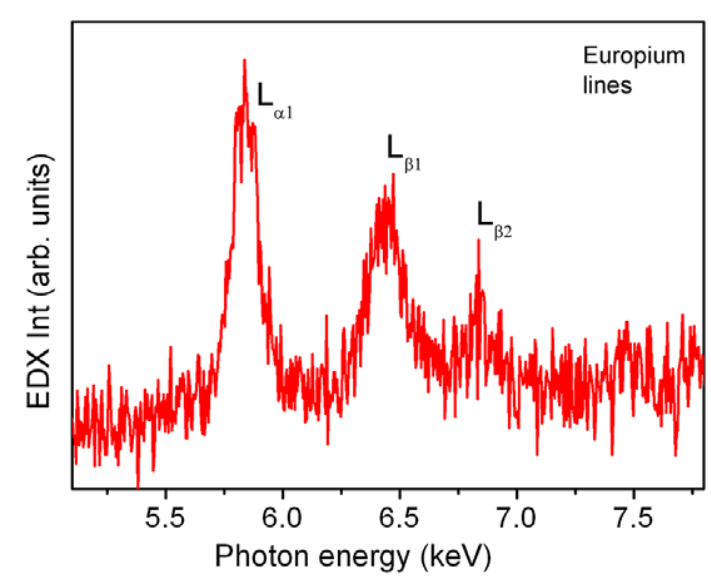

Figure 3. EDX spectrum from nanostructures grown in sample M1.

(EuGG, JCPDS 00-014-0128) in addition to $\beta-\mathrm{Ga}_{2} \mathrm{O}_{3}$. A similar result was previously found in erbium doped gallium oxide starting from a powder mixture [18] in which erbium gallium garnet was detected after the thermal treatment. XRD spectra from samples $\mathrm{D}$ and $\mathrm{R}$ show that only $\beta-\mathrm{Ga}_{2} \mathrm{O}_{3}$ is formed.

Figure 4 shows the normalized CL spectra from pure $\mathrm{Eu}_{2} \mathrm{O}_{3}$ (dashed black), $\beta$ - $\mathrm{Ga}_{2} \mathrm{O}_{3}: \mathrm{Eu}$ in sample $\mathrm{R}$ (solid red) and $\mathrm{Eu}_{3} \mathrm{Ga}_{5} \mathrm{O}_{12}$ in sample $\mathrm{M} 1$ (dotted blue). A red main peak centered at around $610 \mathrm{~nm}$, which corresponds to the

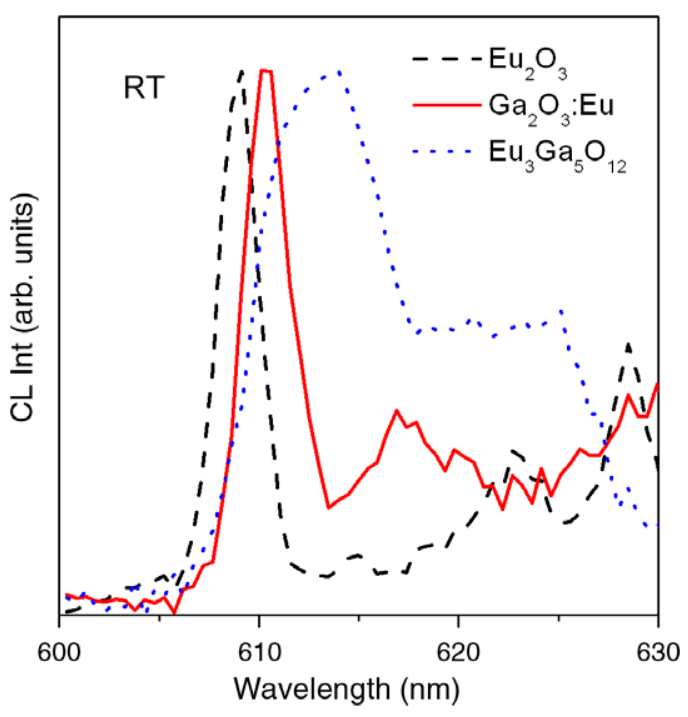

Figure 4. Room temperature $\mathrm{CL}$ due to $\mathrm{Eu}^{3+}$ included in three different hosts: $\mathrm{Eu}_{2} \mathrm{O}_{3}$ (dashed black), $\beta-\mathrm{Ga}_{2} \mathrm{O}_{3}$ (solid red) and $\mathrm{Eu}_{3} \mathrm{Ga}_{5} \mathrm{O}_{12}$ (dotted blue). The spectra have been normalized.

$\mathrm{Eu}^{3+}$ related ${ }^{5} \mathrm{D}_{0}-{ }^{7} \mathrm{~F}_{2}$ intraionic transition, is observed in all of them. However, shifts in the position of the main peak can be observed depending on the host: for $\mathrm{Eu}^{3+}$ in $\mathrm{Eu}_{2} \mathrm{O}_{3}$ is $609 \mathrm{~nm}$, in $\beta-\mathrm{Ga}_{2} \mathrm{O}_{3}$ is $610 \mathrm{~nm}$ and in EuGG is $613 \mathrm{~nm}$. Additionally, to 

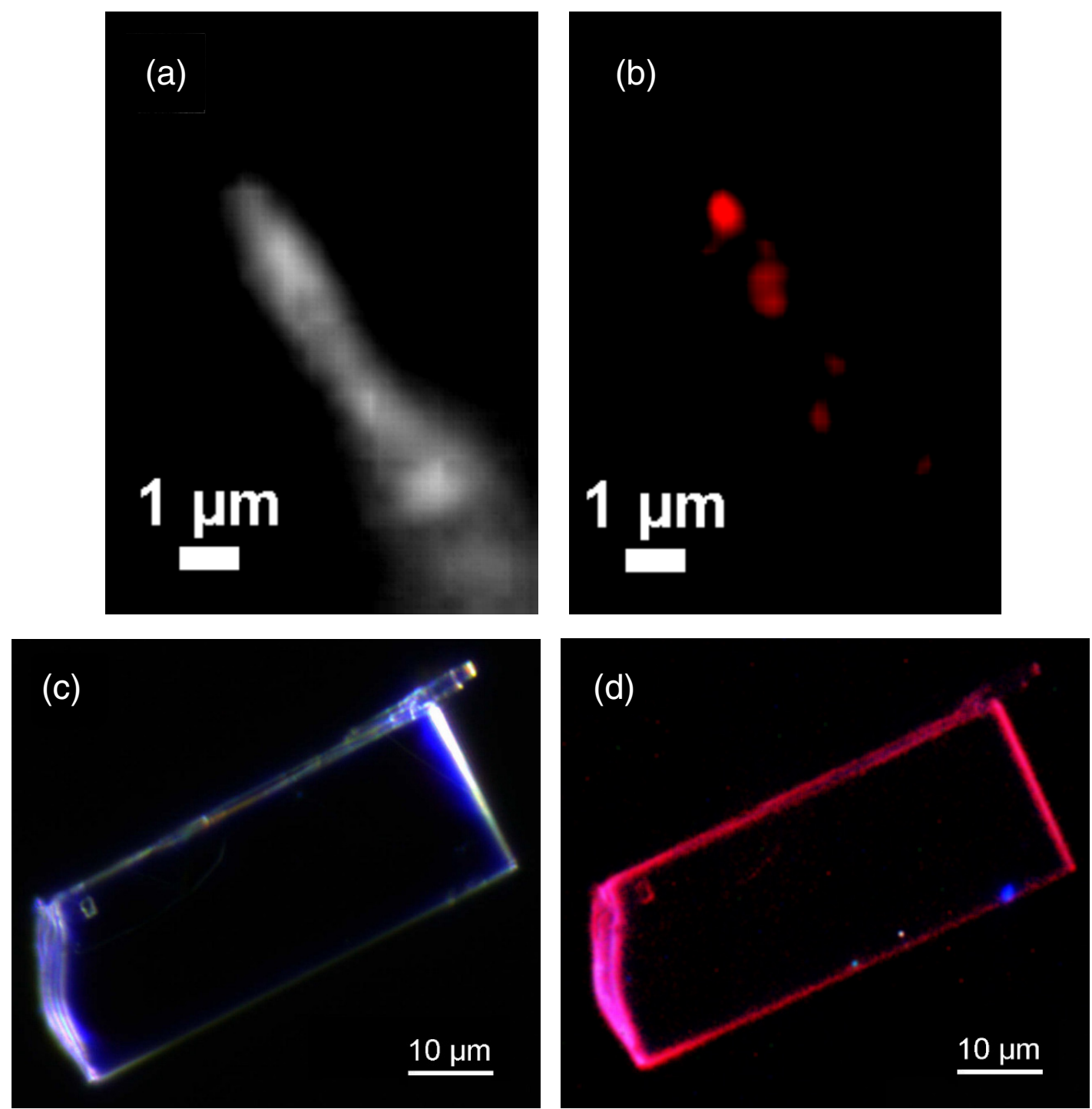

Figure 5. (a), (c) Dark-field optical microscopy and (b), (d) corresponding room temperature $\mu$-PL images of Eu doped structures in sample M1. The $\mu$-PL spectrum in figure 6 was obtained from the structure shown in (d). $\lambda_{\text {exc }}=366 \mathrm{~nm}$.

further differentiate $\mathrm{Eu}$ in $\beta-\mathrm{Ga}_{2} \mathrm{O}_{3}$ and in $\mathrm{Eu}_{2} \mathrm{O}_{3}$, we observe that a peak at around $618 \mathrm{~nm}$ is present in the former, while this second peak is centered at $623 \mathrm{~nm}$ in the latter. Luminescence results from sample M2 are similar to those obtained from sample M1.

It is well known that differences in the crystal field surrounding the rare earth ions result in slight shifts of their luminescence peaks and differences in their relative intensities $[19,20] . \quad \mathrm{Eu}^{3+}$ in $\beta-\mathrm{Ga}_{2} \mathrm{O}_{3}$ is substituting $\mathrm{Ga}$ in octahedral position while in $\mathrm{Eu}_{3} \mathrm{Ga}_{5} \mathrm{O}_{12}$ is within a dodecahedral site with lower symmetry [19]. By comparison of the spectra from different hosts, we can deduce the origin of the luminescence observed in the nanostructures.

The waveguiding behavior of the $\beta-\mathrm{Ga}_{2} \mathrm{O}_{3}$ nanostructures makes them very interesting for potential photonic nanodevices [4]. The nanostructures can guide external light and light emitted by the nanostructures themselves in a photoluminescence process. Figure 5 shows this effect in two different structures obtained in sample M1. The images show the dark- field optical image and the room temperature $\mu$-PL image of two structures. One of them is a nanowire of some hundreds of nanometers diameter, figures 5(a) and (b). The other one, shown in figures 5(c) and (d), is a thin plate as that shown in figure 1(a). Light guiding in both structures leads to the intense light signal observed in their edges or ends. A rough calculation yields a level of confinement of the emitted light of around $80 \%$ by total internal reflection in the microplate. This effect has been used very recently for solar concentrators [21].

Figure 6 shows the room temperature (RT) CL spectra from nanowires in sample D (dotted blue) and from structures in sample M1 (dashed red). The RT $\mu$-PL spectrum obtained from a structure in sample M1 is also plotted (solid black). The spectra show a main peak centered at $610 \mathrm{~nm}$ which, by comparison with figure 4 , is associated to $\mathrm{Eu}^{3+}$ in $\beta-\mathrm{Ga}_{2} \mathrm{O}_{3}$. However, there is a difference between the CL spectra from the structures in D and M1. A broad shoulder at longer wavelengths can be observed in the latter. A contribution from $\mathrm{Eu}^{3+}$ in $\mathrm{Eu}_{3} \mathrm{Ga}_{5} \mathrm{O}_{12}$ clusters formed within the structure could 


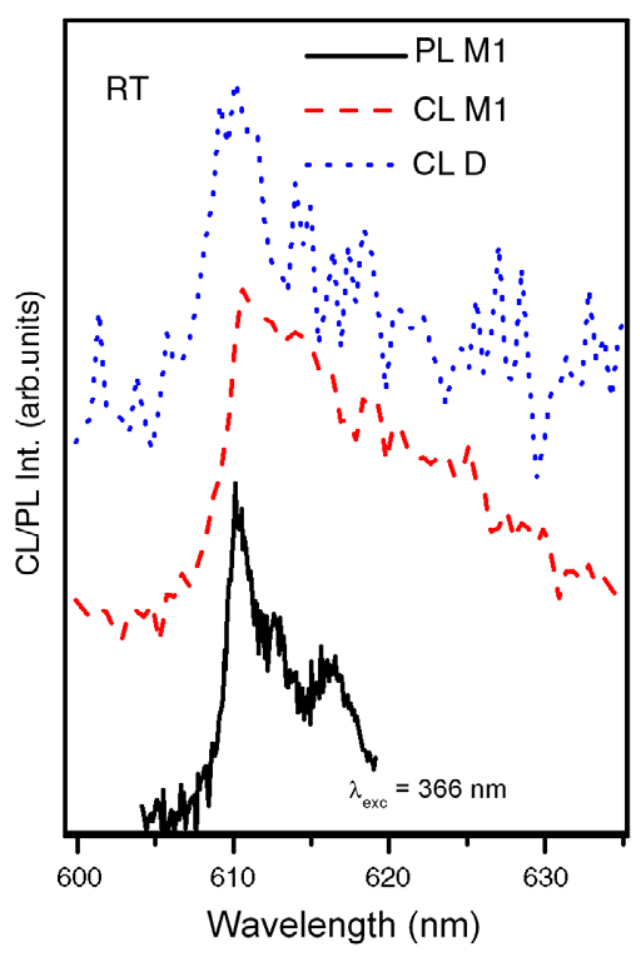

Figure 6. Room temperature CL from samples M1 (dashed red) and $\mathrm{D}$ (dotted blue). Room temperature $\mu$-PL from a microstructure from sample M1 (solid black). The optical and $\mu$-PL images are shown in figures 5(c) and (d). The spectra have been normalized and vertically shifted for the sake of clarity.

explain this result. However, the main peak centered at $610 \mathrm{~nm}$ shows that most of the luminescence is due to $\mathrm{Eu}^{3+}$ in $\beta$ $\mathrm{Ga}_{2} \mathrm{O}_{3}$. The $\mu$-PL spectrum corresponding to the structure from $\mathrm{M} 1$ presents peaks which are associated only to $\mathrm{Eu}^{3+}$ in $\beta-\mathrm{Ga}_{2} \mathrm{O}_{3}$, which shows that the luminescence from these structures is due to europium doped gallium oxide.

In summary, $\mathrm{Eu}$ doped $\beta-\mathrm{Ga}_{2} \mathrm{O}_{3}$ nanowires have been obtained either from a mixture of $\mathrm{Ga}_{2} \mathrm{O}_{3}: \mathrm{Eu}_{2} \mathrm{O}_{3}$ powders or by Eu diffusion into previously obtained nanostructures by high temperature annealing in the presence of $\mathrm{Eu}_{2} \mathrm{O}_{3}$. The morphology of the structures is varied depending on the growth procedure. In particular, a higher number of long, doped nanowires and nanoribbons is obtained by the second method. Europium ions, with a concentration of around 1 at. $\%$ for both growth procedures, have been successfully incorporated into the $\beta-\mathrm{Ga}_{2} \mathrm{O}_{3}$ lattice and behave as optically active centers. Room temperature red luminescence related to the
$\mathrm{Eu}^{3+}$ intraionic transitions has been demonstrated by spatially resolved cathodoluminescence and photoluminescence of the grown structures. Furthermore, $\mu$-PL measurements show that the Eu emitted light is guided through the doped nanowires and nanoplates.

\section{Acknowledgment}

This work has been supported by MEC (Project MAT 200601259).

\section{References}

[1] Huang Y, Duan X F and Lieber C M 2005 Small 1142

[2] Minot E D, Kelkensberg F, van Kouwen M, van Dam J A, Kouwenhoven L P, Zwiller V, Borgstrom M T, Wunnicke O, Verhiejen M A and Bakker E P A M 2007 Nano Lett. 7367

[3] Law M, Sirbuly D J, Johnson J C, Goldberger J, Saykally R and Yang P D 2004 Science 3051269

[4] Nogales E, García J A, Méndez B and Piqueras J 2007 Appl. Phys. Lett. 91133108

[5] Heyden O, Agarwal R and Lieber C M 2006 Nat. Mater. 5352

[6] Tian B, Zheng X L, Kempa T J, Fang Y, Yu N F, Yu G H, Huang J L and Lieber C M 2007 Nature 449885

[7] Lu J C, Chang P and Fan Z 2006 Mater. Sci. Eng. R 5249

[8] Tippins H H 1965 Phys. Rev. A 140316

[9] Yamaga M, Víllora E G, Shimamura K, Ichinose N and Honda M 2003 Phys. Rev. B 68155207

[10] Zhou X T, Heigl F, Ko J Y P, Murphy M W, Zhou J G, Regier T, Blyth R I R and Sham T K 2007 Phys. Rev. B 75125303

[11] Nogales E, Méndez B and Piqueras J 2005 Appl. Phys. Lett. 86113112

[12] Víllora E G, Shimamura K, Ujiie T and Aoki K 2008 Appl. Phys. Lett. 92202120

[13] Binet L and Gourier J 1998 J. Phys. Chem. Solids 591241

[14] Kenyon A J 2005 Semicond. Sci. Technol. 20 R65

[15] Yu D P, Bubendorff J L, Zhou J F, Leprince-Wang Y and Troyon M 2002 Solid State Commun. 124417

[16] Gollakota P, Dhawan A, Wellenius P, Lunardi L M, Muth J F, Saripalli Y N, Peng H Y and Everitt H O 2006 Appl. Phys. Lett. 88221906

[17] Nogales E, García J A, Méndez B and Piqueras J 2007 J. Appl. Phys. 101033517

[18] Nogales E, Méndez B and Piqueras J 2008 Nanotechnology 19035713

[19] Nogales E, García J A, Méndez B, Piqueras J, Lorenz K and Alves E 2008 J. Phys. D: Appl. Phys. 41065406

[20] Dierolf V, Sandman C, Zavada J, Chow P and Hertog B 2004 J. Appl. Phys. 955464

[21] Currie M J, Mapel J K, Heidel T D, Goffri S and Baldo J A 2008 Science $\mathbf{3 2 1} 226$ 